\title{
CLANS WITH ZERO ON AN INTERVAL
}

\author{
BY \\ HASKELL COHEN(1) AND L. I. WADE
}

Following the terminology of Wallace [8] we shall use the word mob to mean a Hausdorff topological semigroup, and shall use clan for a compact connected mob with unit. Interval means a closed interval on the real line, although as A. H. Clifford has pointed out to the authors, nearly all the theorems (and proofs) generalize to arbitrary compact connected linearly ordered topological spaces.

The object of this paper is to characterize clans with zero on an interval. Partial results in this connection have been found by Faucett $[3 ; 4]$ and Clifford [1]. In addition the case when 0 (the zero) is an end point has been studied by Mostert and Shields [5]. Finally a forthcoming paper of Clifford [2] on linear mobs with idempotent endpoints will contain many pertinent results.

In what follows $S$ is always a clan on an interval with zero. It is well known (e.g. Wallace [7]) that the unit $u$ is an end point. We will assume that it is the right hand end point (the other case, of course, can be handled by a dual argument) and call the other end point $\delta$. Let $L$ be the interval $[\delta, 0]$ and $R$ the interval $[0, u]$ so that we have the following diagram for $S$ :

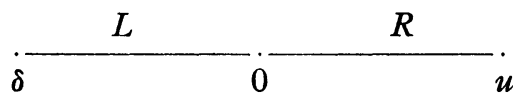

FIG. 1

We define a partial order $<$ on $S$ as follows: $x<y$ if and only if $x$ separates $y$ and 0 , (i.e. $x<y$ if both $x$ and $y$ are on the same side of 0 and $x$ is closer to 0 than $y$ is). We use the notation $l, l_{i} \in L$ and $r, r_{i} \in R$.

The authors would like to express their gratitude to Professor R. J. Koch for suggesting the question considered here as well as for patient listening and helpful suggestions.

1. We look first at the case when $L$ is degenerate. In this case $\delta=0$ and 0 is an endpoint. These clans have been completely determined by the work of Mostert and Shields, and Clifford as noted above. For completeness we include a summary of their results in this section.

Let $S$ be such a clan and let $E=\left\{s \mid s \in S\right.$ and $\left.s^{2}=s\right\}$ (i.e. the idempotents

Presented to the Society, August 23, 1956; received by the editors January 7, 1957.

(1) This research was supported in part by the United States Air Force under Contract No. AF 18(603)-89 monitored by the AF Office of Scientific Research of the Air Research and Development Command. 
in $S$ ). Note that $E$ is closed and consider the closed intervals $[e, f]$ with $e, f \in E$. Let $A=\{[e, f] \mid[e, f]$ is a component of $E\}$ and $B=\{[e, f] \mid(e, f) \cap E$ is empty $\}$. It is easy to see that $S=\cup\{[e, f] \mid[e, f] \in A \cup B\}$. Faucett [3] has established that each $[e, f] \in A \cup B$ is a submob with zero $e$ and unit $f$, and that $[e, f] \neq[g, h]$ and $x \in[e, f], y \in[g, h]$ implies $x y=\min \{x, y\}$. It also follows from Faucett's work that if $x, y \in[e, f] \in A$, then $x y=\min \{x, y\}$. We call such a clan an $M$-mob.

Consider the example (due independently to E. Calabi, A. H. Clifford and A. M. Gleason) of the interval $[1 / 2,1]$ with the multiplication $x \cdot y$ $=\max \{1 / 2, x y\}$. We shall call any mob topologically isomorphic to this example a $C$-mob (regretfully abandoning the euphonious "Calabi mobbi"). It can be shown that if $[e, f] \in B$, it is either topologically isomorphic to the usual unit interval (and will be called a $U$-mob) or is a $C$-mob. Furthermore if $S$ is any interval and $E$ is any closed subset of $S$ containing the endpoints of $S$, then $S$ admits the structure of a clan made up of $U, C$, and $M$ submobs with the minimum multiplication between different submobs and $E$ as the set of idempotents of $S$. Using Clifford's terminology [2], we shall call such a clan "a standard clan."

2. We turn now to the case when 0 is an interior point.

Lemma 2.1. $R$ is a submob.

Proof. Suppose that there are elements $r_{1}$ and $r_{2}$ in $R$ whose product $r_{1} r_{2}=l \in L$. Since multiplication by $r_{1}$ is a continuous function $r_{1} \cdot\left[r_{2}, u\right]$ $\supset\left[r_{1} r_{2}, r_{1} u\right]=\left[l, r_{1}\right]$. Since $0 \in\left[l, r_{1}\right]$ there must exist $r_{3} \geqq r_{2}$ with $r_{1} r_{3}=0$. Again $r_{3}[0, u] \supset\left[0, r_{3}\right]$ which contains $r_{2}$ so that for some $r_{4} \in R$ we have $r_{3} r_{4}=r_{2}$. Now $l=r_{1} r_{2}=r_{1}\left(r_{3} r_{4}\right)=\left(r_{1} r_{3}\right) r_{4}=0 r_{4}=0$ completing the proof.

Note, therefore, that $R$ is a subclan with endpoint 0 so that it is a standard clan.

LEMMA 2.2. $R L \cup L R C L$.

Proof. If, say $r_{1} l=r_{2}$, by an argument similar to that used in the preceding lemma we find $r_{3}$ and $r_{4}$ such that $r_{3} l=0$ and $r_{4} r_{3}=r_{1}$. Now $r_{2}=r_{1} l=\left(r_{4} r_{3}\right) l$ $=r_{4}\left(r_{3} l\right)=0$. Similarly we can show $L R \subset L$.

Lemma 2.3. Either $L^{2} \subset L$ or $L^{2} \subset R$.

Proof. Suppose $l_{1} l_{2}=l$ and $l_{3} l_{4}=r$. We show first that $l_{1}$ and $l_{3}$ may be taken as the same element. If, say $l_{1}>l_{3}$ there is, by continuity, an $r_{1}$ with $l_{3}=l_{1} r_{1} ;$ so, $l_{3} l_{4}=\left(l_{1} r_{1}\right) l_{4}=l_{1}\left(r_{1} l_{4}\right)$. Hence letting $l_{5}=r_{1} l_{4}$ (by Lemma 2.2) we have $l_{1} l_{2}=l$ and $l_{1} l_{5}=r$. Now suppose $l_{2}>l_{5}$. We find $r_{2}$ with $l_{2} r_{2}=l_{5}$ and note that we have $r=l_{1} l_{5}=l_{1}\left(l_{2} r_{2}\right)=\left(l_{1} l_{2}\right) r_{2}=l r_{2}$ which by Lemma 2.2 belongs to $L$. Hence $r$ must be 0 . Note that similar arguments hold when $l_{5}>l_{2}$ and when $l_{3}>l_{1}$. 
Faucett [3] has shown that if $p$ is a cut point of $S$, (i.e., if $S-\{p\}=A \cup B$ with $A$ and $B$ separate) and $K$ (the minimal ideal) $\subset A$, then $p S \cup S p \subset A$. As immediate consequences (since $K=\{0\}$ ) we have

Lemma 2.4. $r_{1} r_{2} \leqq \min \left\{r_{1}, r_{2}\right\}$, lr and $r l \leqq l$, and if $L^{2} \subset L$, then $l_{1} l_{2}$ $\leqq \min \left\{l_{1}, l_{2}\right\}$.

LemmA 2.5. If $r_{1} \prec r_{2}$, then $x r_{1} \leqq x r_{2}$ and $r_{1} x \leqq r_{2} x$ for all $x \in S$. If $l_{1} \prec l_{2}$, then $x l_{1} \leqq x l_{2}$ and $l_{1} x \leqq l_{2} x$ for all $x \in S$.

Proof. For brevity we show one case, all other proofs being similar. If $l_{1} \prec l_{2}$, there is an $r$ such that $l_{2} r=l_{1}$; so $x l_{1}=\left(x l_{2}\right) r$ which is $\leqq x l_{2}$ by Lemma 2.4.

Definition. Two functions $f$ and $g$ on a semigroup are called co-multiplicative if and only if $f\left(r_{1}\right)=g\left(s_{1}\right)$ and $f\left(r_{2}\right)=g\left(s_{2}\right)$ imply $f\left(r_{1} r_{2}\right)=g\left(s_{1} s_{2}\right)$.

LEMMA 2.6. For any $S$ we define f and $g$ from $R$ to $L$ by $f(r)=r \delta$ and $g(r)=\delta r$, then $f$ (and $g$ ) satisfy:

(i) $f(0)=0$ and $f(u)=\delta$,

(ii) $f$ is monotone (i.e. $r_{1}>r_{2}$ implies $f\left(r_{1}\right) \geqq f\left(r_{2}\right)$ ),

(iii) $f$ is continuous,

(iv) If $t_{0}$ is the zero of $T, a C$ or $U$-submob of $R$, then $t_{1}$ and $t_{2} \in T$ and $f\left(t_{1}\right)=f\left(t_{2}\right)$ imply $t_{1}=t_{2}$ or $f\left(t_{1}\right)=f\left(t_{0}\right)$,

(v) $f$ and $g$ are co-multiplicative.

Proof. The first three statements are obvious, and for (iv) if say $t_{1}>t_{2}$, there is a $t_{3} \in T$ such that $t_{3} t_{1}=t_{2}$. Now $t_{1} \delta=f\left(t_{1}\right)=f\left(t_{2}\right)=t_{2} \delta=t_{3} t_{1} \delta$. Therefore $t_{1} \delta=t_{3} t_{1} \delta=t_{3}^{2} t_{1} \delta=\cdots=t_{3}^{n} t_{1} \delta$. The sequence $\left\{t_{3}^{n}\right\}$ converges to $t_{0}$, so by continuity $t_{1} \delta=t_{0} t_{1} \delta=t_{0} \delta$, and $f\left(t_{1}\right)=f\left(t_{0}\right)$.

(v) Suppose $f\left(r_{1}\right)=g\left(s_{1}\right)$ and $f\left(r_{2}\right)=g\left(s_{2}\right)$; then $f\left(r_{1} r_{2}\right)=\left(r_{1} r_{2}\right) \delta=r_{1}\left(r_{2} \delta\right)$ $=r_{1} f\left(r_{2}\right)=r_{1} g\left(s_{2}\right)=r_{1} \delta s_{2}=\left[f\left(r_{1}\right)\right] s_{2}=\left[g\left(s_{1}\right)\right] s_{2}=\delta s_{1} s_{2}=g\left(s_{1} s_{2}\right)$.

3. All standard clans are abelian. Thus $R$ is always abelian. We shall show below that $L$ is abelian; hence, if $S$ fails to be abelian, this failure must occur among the mixed products (elements of $L$ multiplied by elements of $R$ ). We offer such an example. Consider the interval from -1 to 1 with multiplication "." as follows:

For $s$ and $t$ non-negative $s \cdot t=s t$ (the usual product)

$$
(-s) \cdot(-t)=0, \quad(-s) \cdot t=-(s t), \quad s \cdot(-t)=-\left(s^{2} t\right) .
$$

Note that $L^{2}=0$ in the example. We call such clans left trivial and devote this section to their study.

Construction 3.1. Let $S=[\delta, u]$ be any interval, and 0 any point in the open interval $(\delta, u)$. Let $R=[0, u]$ and $L=[\delta, 0]$, and define a multiplication "." on $R$ making it into any standard clan. Let $f$ and $g$ be any two functions 
on $R$ to $L$ satisfying (i) through (v) of Lemma 2.6. Define a multiplication "०" on $S$ as follows:

$$
\begin{array}{ll}
r_{1} \circ r_{2}=r_{1} \cdot r_{2}, & r \circ l=f\left(r \cdot f^{-1}(l)\right), \\
l_{1} \circ l_{2}=0, & l \circ r=g\left(r \cdot g^{-1}(l)\right) .
\end{array}
$$

TheOREM 3.2. $(S, \mathrm{o})$ is a left trivial clan, and any left trivial clan can be so constructed.

This theorem will be proved by a sequence of lemmas.

Lemma 3.3. $r \circ l$ (and $l \circ r$ ) are well defined.

Proof. Let $k \in f^{-1}(l)$ and $m=\inf f^{-1}(l)$. If $k \neq m$, by (iv) of Lemma 2.6 $m^{2}=m$. If $r k \neq r m, r>m$; so $r m=m$, and we have $f(r k) \leqq f(k)=f(m)=f(r m)$ $\leqq f(r k)$. Thus $f(r k)=f(r m)$ as was to be shown.

Note that we have shown that in the definition of "O" any element of $f^{-1}(l)\left[\right.$ or $\left.g^{-1}(l)\right]$ may be selected. As consequences we have

REMARK 1. $f\left(r \cdot f^{-1} f(t)\right)=f(r \cdot t)$ and $g\left(r \cdot g^{-1} g(t)\right)=g(r \cdot t)$ for all $r, t \in R$.

REMARK 2. $f(r)=f(r \cdot u)=r \circ \delta$ and $g(r)=\delta \circ r$.

Lemma 3.4. "O" is continuous.

Proof. Suppose $r \circ l=a \in(\delta, 0)$. We prove this case only; the modifications for the other cases being tedious but obvious. Suppose $N$ is any neighborhood of $a$. We need to find $U$ and $W$, neighborhoods of $r$ and $l$ respectively with $U \circ W \subset N$. Since $f$ is continuous $f^{-1}(N)$ is open, and by continuity of multiplication in $R$, we have open sets $U$ and $V$ containing $r$ and $f^{-1}(l)$ with $U \cdot V \subset f^{-1}(N)$. Hence $f(U \cdot V) \subset N$. Let $p \in V$ with $p<m=\inf f^{-1}(l)$; then $f(p)<l$ and $\sup f^{-1} f(p)<m$. Pick $q$ so that $\sup f^{-1} f(p)<q<m$, then $f(p)<f(q)$ $\prec l$. In a similar manner we can find $k$ so that $k>\sup f^{-1}(l)$ and $f^{-1} f(k) \subset V$. Now let $W$ be the open interval $(f(k), f(q)) ; l \in W$ and $U \circ W=f\left(U \cdot f^{-1}(W)\right)$ $\subset f(U \cdot V) \subset N$.

Lemma 3.5. For $r, s \in R$ and $l \in L, r \circ(s \circ l)=(r \cdot s) \circ l\lceil$ and dually $l \circ(r \cdot s)$ $=(l \circ r) \circ s]$.

Proof. $r \circ(s \circ l)=f\left(r \cdot f^{-1}(s \circ l)\right)=f\left(r \cdot f^{-1} f\left(s \cdot f^{-1}(l)\right)\right)=f\left(r \cdot\left(s \cdot f^{-1}(l)\right)\right)$ $=(r \cdot s) \circ l$.

LemaA 3.6. $f$ and $g$ co-multiplicative implies $(r \circ \delta) \circ s=r \circ(\delta \circ s)$.

Proof. Let $r, s \in R$; then, there exist $r^{1}$ and $s^{1} \in R$ with $f(r)=g\left(r^{1}\right)$ and $g(s)=f\left(s^{1}\right)$. Now $(r \circ \delta) \circ s=g\left(s \cdot g^{-1} f(r)\right)=g\left(s \cdot g^{-1} g\left(r^{1}\right)\right)=g\left(s \cdot r^{1}\right)=f\left(s^{1} \cdot r\right)$ $=f\left(r \cdot s^{1}\right)=f\left(r \cdot f^{-1} f\left(s^{1}\right)\right)=f\left(r \cdot f^{-1} g(s)\right)=r \circ(\delta \circ s)$.

Lemma 3.7. $(r \circ \delta) \circ s=r \circ(\delta \circ s)$ for all $r$ and $s \in R$ implies $(r \circ l) \circ s$ $=r \circ(l \circ s)$ for all $r$ and $s$ in $R$ and $l \in L$. 
Proof. For $l \in L$ there are $p$ and $q \in R$ with $l=f(p)=g(q)$. So $l=p \circ \delta$ and $\delta \circ q$. Thus $(r \circ l) \circ s=(r \circ(\delta \circ q)) \circ s=((r \circ \delta) \circ q) \circ s=(r \circ \delta) \circ(q \cdot s)$ $=r \circ(\delta \circ(q \cdot s))=r \circ((\delta \circ q) \circ s)=r \circ(l \circ s)$.

Proof of Theorem 3.2. That "O" is associative follows from Lemmas 3.5 and 3.7. Clearly $u$ is a unit for $S$ and $S$ is left trivial. Conversely if $S$ is any left trivial clan, Lemma 2.6 insures that the functions $f$ and $g$ (defined in that Lemma) have the desired properties, and it is easy to see that the construction recreates the original multiplication in $S$.

\section{Lemma 4.1. For each $r \in R, \delta^{2} r=\delta r \delta=r \delta^{2}$.}

Proof. Let $r \in R$. If $r \delta=\delta r$, the conclusion is immediate; suppose, then $\delta r\langle r \delta$ (an analogous argument holds if $\delta r\rangle r \delta$ ).

CASE 1. $\delta^{2} \in R$. Using 2.5 we get $\delta^{2} r \leqq \delta r \delta$ and $\delta r \delta \leqq r \delta^{2}$. Now $\delta^{2} \in R$ means $\delta^{2} r=r \delta^{2}$ and the conclusion follows.

CASE 2. $\delta^{2} \in L$. There is a $k \in R$ such that $k r \delta=\delta r$, and also there is $p \in R$ with $p \delta=\delta^{2}$. Using these relations and the fact that $R$ is commutative, we get $\delta r \delta=k r \delta^{2}=k r p \delta=p k r \delta=p \delta r=\delta^{2} r$. Now since $r \delta>\delta r$, there is $s \in R$ with $s>r$ and $r \delta=\delta s$. Since $s>r, s \delta \geqq r \delta=\delta s$; so that by an argument as above we get $\delta s \delta=\delta^{2} s$. Now $r \delta^{2}=(r \delta) \delta=\delta s \delta=\delta^{2} s=\delta(\delta s)=\delta r \delta$ completing the proof.

LEMma 4.2. $L$ is abelian.

Proof. For $l_{1}$ and $l_{2} \in L$ there are $r_{1}$ and $r_{2} \in R$ with $\delta r_{i}=l_{i}$. Therefore, using 4.1 and the commutativity of $R$ we have $l_{1} l_{2}=\delta r_{1} \delta r_{2}=\delta^{2} r_{1} r_{2}=\delta^{2} r_{2} r_{1}=\delta r_{2} \delta r_{1}$ $=l_{2} l_{1}$.

Definition. We will call a clan pointed if in it $l^{2}=\delta^{2}$ implies $l=\delta$.

Theorem 4.3. A pointed clan is abelian.

Proof. Since $R$ and $L$ are each commutative and each $l=r \delta$ for some $r$, we need only show $r \delta=\delta r$ for all $r$. We divide the proof into three parts.

CASE 1. If $\delta^{2}$ is idempotent, then $\left(\delta^{3}\right)^{2}=\left(\delta^{2}\right)^{3}=\delta^{2}$. Hence by pointedness $\delta^{3}=\delta$ and $\delta^{2}$ is a unit for $\delta$. Then using 4.1 we have $r \delta=r \delta^{2} \delta=\delta r \delta \delta=\delta \delta \delta r=\delta r$.

CASE $2\left(^{2}\right)$. If $\delta^{2} \in R$ and is not idempotent, $\delta^{2} \in[z, p]$, a $C$ or $U$-mob, and there is $s \in(z, p)$ with $z \prec s \delta^{2} \prec \delta^{2}$. If $\delta s \prec s \delta$, there is $t>s$ with $\delta t=s \delta$ and $s \delta^{2}=\delta t \delta=t \delta^{2}$. If $t \geqq p$, then $t \delta^{2}=\delta^{2}$, but $t \delta^{2}=s \delta^{2}<\delta^{2}$. Hence $s<t<p$, and $z<s \delta^{2}=t \delta^{2}$ implies $s=t$. Since a similar argument holds if $s \delta<\delta s$ we have $\delta s=s \delta$. Now if $r \in(z, p)$, there is an $s$ as above and a positive integer $n$ such that $s^{n}=r$. Since $s$ commutes with $\delta$, so does $r$. If $r \geqq p,(r \delta)^{2}=r \delta r \delta=r^{2} \delta^{2}=\delta^{2}$ so $r \delta=\delta$ and similarly $\delta r=\delta$ so $r \delta=\delta r$. Finally if $r \leqq z, r \delta=\left(r \delta^{2}\right) \delta=\delta r \delta^{2}=\delta r$.

CASE 3. If $\delta^{2} \in L$ and $\delta^{2}$ is not idempotent, let $p$ and $q \in R$ be such that $p \delta=\delta^{2}=\delta q$ and suppose $p>q$. If $f^{2}=f \in[q, p]$, then $f \geqq q$ implies $\delta^{2}=\delta q=\delta q f$ $=\delta^{2} f=\delta^{2} f^{2}=(f \delta)^{2}$ and $f \delta=\delta$. Now $f \leqq p$ implies $\delta^{2}=p \delta \geqq f \delta=\delta$, so that $\delta^{2}=\delta$, here.

(2) The authors express their thanks to the referee for the shortened proof of Case 2 given 
a contradiction. Therefore $p$ and $q$ are nonidempotent elements of $[z, e]$, a $C$ - or $U$-mob, and hence have unique $n$th roots in $[z, e]$. If, say, $p^{1 / n} \delta>\delta q^{1 / n}$, there is $r \in(z, e)$ with $\delta q^{1 / n}=r p^{1 / n} \delta$. Multiplying on the right by $q^{(n-1) / n}$ gives $\delta q=r p^{1 / n} \delta q^{(n-1) / n}=r p^{1 / n} r p^{1 / n} \delta q^{(n-2) / n}=\cdots=r^{n} p \delta=r^{n} \delta q$; hence $\delta^{2}=\delta q=r^{n} \delta q$ $=r^{n} \delta^{2}=r^{2 n} \delta^{2}=\cdots=z \delta^{2} q \leqq 2 \delta^{2}=\delta^{4}$. This contradiction shows $p^{1 / n} \delta=\delta q^{1 / n}$. Since $p^{1 / n}$ approaches $e$ and $e \delta=\delta$, there is an integer $m$ with $\left(p^{1 / m} \delta\right)^{2}>\delta^{4}$. Now if $q=p r$ for some $r \in(z, e),\left(p^{1 / m} \delta\right)^{2}=\left(\delta q^{1 / m}\right)^{2}=\left(\delta p^{1 / m} r^{1 / m}\right)^{2}=r^{2 / m}\left(p^{1 / m} \delta\right)^{2}$ (by 4.1). Hence, $\left(p^{1 / m} \delta\right)^{2}=r^{2 / m}\left(p^{1 / m} \delta\right)^{2}=r^{4 / m}\left(p^{1 / m} \delta\right)^{2}=z\left(p^{1 / m} \delta\right)^{2} \leqq z \delta^{2} \leqq p^{2} \delta^{2}=\delta^{4}$. This contradiction shows $p=q$ and $p^{1 / n} \delta=\delta p^{1 / n}$. Therefore $p^{m / n} \delta=\delta p^{m / n}$ (where $p^{m / n}$ is defined to be $\left.\left(p^{1 / n}\right)^{m}\right)$ and since the rational powers of $p$ are dense in $[z, e], r \delta=\delta r$ for all $r \in[z, e]$. As in Case 2, if $r>e,(r \delta)^{2}=(r(e \delta))$ $=(e \delta)^{2}=\delta^{2}=(\delta e)^{2}=(\delta e r)^{2}=(\delta r)^{2}$ and pointedness shows $r \delta=\delta=\delta r$. Finally if $r<z, r \delta=r p \delta=r \delta^{2}=\delta^{2} r=\delta p r=\delta r$.

5. In this section we examine clans with $R$ a $C$ or $U$-mob. Since we have done the left trivial case in $\$ 3$, we make the (sometimes tacit) assumption throughout this section that $\delta^{2} \neq 0$.

Lemma 5.1. If $S$ is a clan with $\delta^{2} \neq 0$ and $R$ a $C$ or $U$-mob, then $S$ is pointed and hence abelian.

Proof. If $l^{2}=\delta^{2}$ with $l=r \delta$ and $r<u$, then $\delta^{2}=(r \delta r \delta)=r^{2} \delta^{2}=r^{4} \delta^{2}=\cdots=0$, a contradiction.

Consider now the interval from -1 to 1 . It is possible to make this into a clan with $L^{2} \subset R$ by using ordinary multiplication. It is also possible to have $L^{2} C L$ by defining products of negative numbers to be negative (more precisely define "O" on $[-1,1]$ by

$$
\begin{aligned}
& x \circ y=0 \text { if } x \text { or } y=0, \\
& x \circ y=\min \left[\frac{x}{|x|}, \frac{y}{|y|}\right]|x y| \text { otherwise). }
\end{aligned}
$$

We adopt the notation $[-a, 1]$ means the interval from $-a$ to 1 with ordinary multiplication and $[-a, 1]_{N}$ means the interval with the "negative" multiplication.

Lemma 5.2. If $S$ is a clan with $\delta^{2} \in R$ a U-mob, then $S$ is topologically isomorphic to $[-a, 1]$ for some $a$.

Proof. Since $R$ is a $U$-mob, we have $f$ a topological isomorphism on $[0,1]$ to $R$. Let $p \in R$ be such that $p^{2}=\delta^{2}$. Let $a=f^{-1}(p)$. Define $g:[-a, 1] \rightarrow S$ as follows:

$$
\begin{aligned}
& \text { For } t \in[0,1], g(t)=f(t), \\
& \text { For } t \in[-a, 0], g(t)=f(-t) \delta / p .
\end{aligned}
$$

It is straightforward to verify that $g$ is a topological isomorphism onto. 
Lemma 5.3. If $S$ is a clan with $\delta^{2} \in L$ and $R$ is a $U$-mob, then $S$ is topologically isomorphic to $[-a, 1]_{N} /[-b, 0]$.

Proof. As in 5.2 we have $f:[0,1] \rightarrow R$. Let $p \in R$ such that $p \delta=\delta^{2}$. Let $a=f^{-1}(p)$. Let $m=\sup \left\{l \mid l^{2}=0\right\}, q=\sup \{r \mid r m=0\}$, and $b=f^{-1}\left(q^{2}\right)$. We show first that $a>b$. Let $s=\sup \{r \mid r \delta=m\}$; then $s^{1 / 2} \delta>m$ and, hence, $s \delta^{2}>0$. Now $p m=p s \delta=s p \delta=s \delta^{2}>0$; therefore $p>q>q^{2}$ and $a>b$. Now define $g:[-a, 1]_{N} /[-b, 0] \rightarrow S$ by

$$
\begin{aligned}
& \text { For } \quad t \in[0,1], g(t)=f(t), \\
& \text { For } t \in[-a,-b], g(t)=f(-t) \delta / p .
\end{aligned}
$$

We verify that $g(-b)=0$. By definition $g(-b)=[f(b) / p] \delta=\left[q^{2} / p\right] \delta=q(q / p) \delta$. If $(q / p) \delta>m, q / p>s$, and $(s q / p)^{1 / 2}>s ;$ but $\left((s q / p)^{1 / 2} \delta\right)^{2}=[(s q) / p] \delta^{2}=s q \delta=q m$ $=0$. Therefore $(q / p) \delta \leqq m$ and $g(-b)=q(q / p) \delta=0$. We show now that $g$ is 1 to 1 . We need only consider the case $\left[f\left(-t_{1}\right) / p\right] \delta=\left[f\left(-t_{2}\right) / p\right] \delta$. By (iv) of Lemma 2.6 either $f\left(-t_{1}\right) / p=f\left(-t_{2}\right) / p$ and $t_{1}=t_{2}$, or $\left[f\left(-t_{1}\right) / p\right] \delta=0$. Now $s \delta=m \geqq 0$ implies the existence in $R$ of $k$ such that $k s=f\left(-t_{1}\right) / p$. Since $k s \delta=0$, $k m=0$ and $k \leqq q$. Therefore $f\left(-t_{1}\right) / p \leqq q s$ and $f\left(-t_{1}\right) \leqq q p s$. Now $p s m=p s s \delta$ $=s^{2} \delta^{2}=(s \delta)^{2}=m^{2}=0$; so $p s \leqq q, f\left(-t_{1}\right) \leqq q^{2}$, and $-t_{1} \leqq b$. Since the points in $[-b, 0]$ have been identified, $t_{1}$ (and similarly $\left.t_{2}\right)=b$. The remaining properties are easy to verify and we leave them to the reader.

Lemma 5.4. If $S$ is a clan with $\delta^{2} \in R, a C$-mob, then $S$ is topologically isomorphic to some $[-a, 1] /[-b, 1 / 2]$.

Proof. Since $R$ is a $C$-mob, there is $f$, a topological isomorphism on $[1 / 2,1] \rightarrow R$. To simplify the notation involved we consider the elements of the $C$-mob $[1 / 2,1]$ as a subset of the $U$-mob $[0,1]$. We use "." to signify $C$-mob multiplication, "o" for the usual multiplication, and juxtaposition for multiplication in $S$. Define $h: R \rightarrow[0,1]$ by $h(r)=f^{-1}(r)$.

Let $p \in R$ be such that $p^{2}=\delta^{2}$,

$$
\begin{aligned}
q & =p^{1 / 2}, & & a=h(q) \circ h(q), \\
w & =\sup \left\{r \mid r^{2} \delta=0\right\}, & b & =h(q) \circ h(q) \circ h(w) \circ h(w) .
\end{aligned}
$$

It is easy to verify that $[-b, 1 / 2]$ is an ideal of $[-a, 1]$. We form $T$ $=[-a, 1] /[-b, 1 / 2]$, use "." to indicate multiplication in $T$, and consider $T$ as a subset of $[-a, 1]$. We define $g: T \rightarrow S$ by

$$
\begin{aligned}
& g(t)=f(t) \text { if } t \geqq 1 / 2, \\
& g(t)=\left[f\left((-t)^{1 / 2}\right) / q\right]^{2} \delta \text { if } t \in[-a,-b] .
\end{aligned}
$$

To show $g$ is well defined, and continuous we verify

(i) $t \in[-a,-b]$ implies $(-t)^{1 / 2} \geqq 1 / 2$.

(ii) $t \in[-a,-b]$ implies $0<f\left((-t)^{1 / 2}\right) \leqq q$.

(iii) $g(-b)=g(1 / 2)=0$. 
For (i) $t \in[-a,-b]$ means $-t \geqq b$ and $(-t)^{1 / 2} \geqq b^{1 / 2}=h(q) \circ h(w)$ $\geqq \min \{h(g) \circ h(q), h(w) \circ h(w)\}$. Now $q^{2}=p$ means $h\left(q^{2}\right)=h(p) \geqq 1 / 2$; so that $h\left(q^{2}\right)=h(q) \circ h(q)$. Similarly $w^{2} \geqq 0$ and $h(w) \circ h(w)=h\left(w^{2}\right)$. Hence min $\{h(q) \circ h(q), h(w) \circ h(w)\}=\min \left\{h\left(q^{2}\right), h\left(w^{2}\right)\right\} \geqq 1 / 2$ and (i) is established.

(ii) Note first $p \neq 0$; hence if $w q=0, w<q$ and $(w q)^{1 / 2}>w$; but $\left((w q)^{1 / 2}\right)^{2} \delta$ $=w q \delta=0$ contradicting the maximality of $w$. Thus $w q>0$ and $f\left((-t)^{1 / 2}\right)$ $\geqq f\left(b^{1 / 2}\right)=f(h(q) \circ h(w))=f(h(q) \cdot h(w))=q w>0$. Also $f\left((-t)^{1 / 2}\right) \leqq f\left(a^{1 / 2}\right)$ $=f(h(q))=q$.

$$
\begin{aligned}
g(-b) & =\left[\frac{f\left(b^{1 / 2}\right)}{q}\right]^{2} \delta=\left[\frac{f(h(q) \cdot h(w))}{q}\right]^{2} \delta=\left[\frac{q w}{q}\right]^{2} \delta, \\
& =w^{2} \delta=0=f\left[\frac{1}{2}\right]=g\left[\frac{1}{2}\right] .
\end{aligned}
$$

To show $g$ is $1-1$ we first show $t \notin[-b, 1 / 2]$ implies $g(t) \neq 0$. The case $t>1 / 2$ is trivial to show, and $-t \in[a, b)$ implies $f\left[(-t)^{1 / 2}\right]>f(b)=q w$. So that $f\left[(-t)^{1 / 2}\right] / q>w$ and $\left[f\left[(-t)^{1 / 2}\right] / q\right]^{2} \delta$ cannot be zero. Now repeating the argument in the previous lemma shows $g$ is $1-1$.

To show $g$ is a homomorphism we verify three cases

(i) $t_{1}, t_{2} \in[1 / 2,1]$. Then $g\left(t_{1} \cdot t_{2}\right)=f\left(t_{1} \cdot t_{2}\right)=f\left(t_{1}\right) f\left(t_{2}\right)=g\left(t_{1}\right) g\left(t_{2}\right)$.

(ii) $t_{1} \in[1 / 2,1], t_{2} \in[-a,-b]$. Then $-\left(t_{1} \cdot t_{2}\right)=\max \left\{b, t_{1} \circ\left(-t_{2}\right)\right\}$ and $f\left(\left(-\left(t_{1} \cdot t_{2}\right)\right)^{1 / 2}\right)=f\left[\max \left\{b^{1 / 2},\left(t_{1} \circ\left(-t_{2}\right)\right)^{1 / 2}\right\}\right]=f\left[\max \left\{b^{1 / 2},\left(t_{1} \cdot\left(-t_{2}\right)\right)^{1 / 2}\right\}\right]$ $=\max \left\{f\left(b^{1 / 2}\right), f\left(\left(t_{1} \cdot\left(-t_{2}\right)\right)^{1 / 2}\right)\right\}$. Hence

$$
\begin{aligned}
g\left(l_{1} \cdot l_{2}\right) & =\left[\frac{\max \left\{f\left(b^{1 / 2}\right), f\left(\left(t_{1} \cdot\left(-t_{2}\right)\right)^{1 / 2}\right\}\right.}{q}\right]^{2} \delta \\
& =\max \left\{\left[\frac{f\left((b)^{1 / 2}\right)}{q}\right]^{2} \delta,\left[\frac{f\left(\left(t_{1} \cdot\left(-t_{2}\right)\right)^{1 / 2}\right)}{q}\right]^{2} \delta\right\} \\
& =\max \left\{0,\left[\frac{f\left(\left(t_{1}\right)^{1 / 2}\right) f\left(\left(-t_{2}\right)^{1 / 2}\right)}{q}\right]^{2} \delta\right\} \\
& =f\left(t_{1}\right)\left[\frac{f\left(\left(-t_{2}\right)^{1 / 2}\right)}{q}\right]^{2} \delta=g\left(t_{1}\right) g\left(t_{2}\right) .
\end{aligned}
$$

(iii) $t_{1}, t_{2} \in[-a,-b]$. Then

$$
\begin{aligned}
g\left(t_{1}\right) g\left(t_{2}\right) & =\left[\frac{f\left(\left(-t_{1}\right)^{1 / 2}\right)}{q}\right]^{2} \delta\left[\frac{f\left(\left(-t_{2}\right)^{1 / 2}\right)}{q}\right]^{2} \delta=\left[\frac{f\left(\left(-t_{1}\right)^{1 / 2}\right)}{q} \frac{f\left(\left(-t_{2}\right)^{1 / 2}\right) p}{q}\right]^{2} \\
& =\left[f\left(\left(-t_{1}\right)^{1 / 2}\right) f\left(\left(-t_{2}\right)^{1 / 2}\right)\right]^{2}=f\left(\left(-t_{1}\right)^{1 / 2} \cdot\left(-t_{1}\right)^{1 / 2} \cdot\left(-t_{2}\right)^{1 / 2} \cdot\left(-t_{2}\right)^{1 / 2}\right) \\
& =f\left(\max \left\{\frac{1}{2},\left(-t_{1}\right) \circ\left(-t_{2}\right)\right\}\right)=f\left(\max \left\{\frac{1}{2}, t_{1} \circ t_{2}\right\}\right) \\
& =f\left(t_{1} \cdot t_{2}\right)=g\left(t_{1} \cdot t_{2}\right),
\end{aligned}
$$

and the proof is complete. 
Lemma 5.5. If $S$ is a clan with $R$ a $C$-mob and $\delta^{2} \in L$, then $S$ is topologically isomorphic to some $[-a, 1]_{N} /[-b, 1 / 2]$.

Proof. As in the previous lemma we have $f:[1 / 2,1] \rightarrow R$. Consider $\tilde{S}$ which consists of $S$ with $R$ replaced by $[0,1]$ (identifying the "zero"). Let $L$ retain its multiplication from $S,[0,1]$ have its usual products, and define $t \cdot l=l \cdot t=f(t) l$ if $t \geqq 1 / 2$ and 0 otherwise. It is easy to see that $\tilde{S}$ is a clan, and moreover one satisfying the hypotheses of Lemma 5.3. Hence we have $a, b$, and $g$ satisfying $g:[-a, 1]_{N} /[-b, 0] \rightarrow \widetilde{S}$ is a topological isomorphism; moreover, we take $g$ to be the identity on $[0,1]$. If we now define $\tilde{f}: \widetilde{S} \rightarrow S$ by $\tilde{f}(l)=l, \tilde{f}(t)=f(t)$ if $t \geqq 1 / 2$, and $\tilde{f}(t)=0$ otherwise, and let $\eta:[-a, 1]_{N} /[-b, 0]$ $\rightarrow[-a, 1]_{N} /[-b, 1 / 2]$ be the natural homomorphism; we have the following diagram:

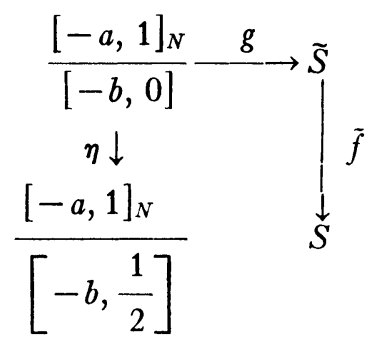

Now by standard arguments there is induced $g^{*}:[-a, 1]_{N} /[-b, 1 / 2] \rightarrow S$ (where $g^{*}=\tilde{f} g \eta^{-1}$ ) which has the required properties.

Definition. A base clan is one topologically isomorphic to $[-a, 1] / I$ (where $0 \prec a \leqq 1$ and $I$ is any closed ideal of $[-a, 1]$ ).

An $N$-base clan is one topologically isomorphic to $[-a, 1]_{N} / I$ ( $I$ any closed ideal of $[-a, 1]_{N}$ ).

The results in the previous lemmas may now be summarized and restated as

TheOREm 5.6. If $S$ is a clan with $\delta^{2} \neq 0$ and $R$ a $C$-or $U$-mob, then $S$ is a base clan or an $N$-base clan. val.

6. In this section we characterize the general clan with zero on an inter-

Definition. A clan $S$ will be called full if either $\delta^{2}=\delta$ or $\delta^{2}=u$. Note that a full clan is pointed, and hence abelian by Theorem 4.3.

Definition. Two or more clans will be called matched if all have $L^{2} \subset L$ or all have $L^{2} \subset R$.

Definition. A clan $S$ will be called an extension of $B$ by $A$ if:

(i) $A$ is a clan,

(ii) $B$ is an ideal and a subclan of $S$, and

(iii) $A=S / B$. 
Suppose $S$ is an extension of $B$ by $A$. We examine the structure of $S$. Let $A=\left[\delta_{A}, u_{A}\right]$ and $B=\left[\delta_{B}, u_{B}\right]$. Now in $S$, since $B$ is a subclan, it must be connected. Since $B$ is an ideal, it must contain the zero. Since $A$ is a nontrivial quotient it must contain the (image of the) unit, and finally if $A$ is not standard $\delta_{A}$ is (the image of) $\delta$ (of $S$ ). So that $S$, in general, may be represented by the diagram:

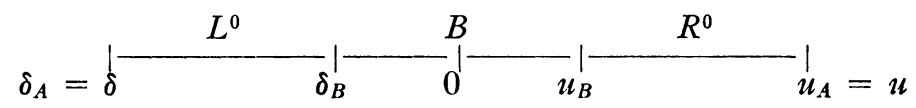

FIG. 2

where $L^{0}$, and $R^{0}$ stand for the half open intervals $\left[\delta_{A}, 0_{A}\right)$ and $\left(0_{A}, u_{A}\right]$ respectively.

Lemma $6.1\left(^{3}\right)$. Let $A$ and $B$ be clans. Then there is a unique extension of $B$ by $A$ if and only if

(i) $A$ and $B$ are matched and,

(ii) Either $B$ is full, $A$ is standard, or $A$ is left trivial.

Proof. "Only if": If $\delta_{A}^{2}=0_{A}, A$ is matched with any clan. If not $\delta_{A}^{2} \in\left(L^{0} \cup R^{0}\right)$ and $A$ and $B$ are matched by 2.3. If $A$ is not standard, $\delta_{A}>\delta_{B}$. If $A$ is not left trivial, $\delta_{A}^{2} \in\left(L^{0} \cup R^{0}\right)$. Let $x \in R^{0}$ and $y \in L^{0}$. Since $B$ is an ideal, $x u_{B}$ and $y u_{B} \in B$. But $x u_{B} \geqq u_{B} u_{B}=u_{B}$, and $y u_{B}>\delta_{B} u_{B}=\delta_{B}$. Thus $x u_{B}=u_{B}$ and $y u_{B}$ $=\delta_{B}$ and $\delta_{B}$ may be written as $\delta_{A} u_{B}$ and $\delta_{B}^{2}=\left(\delta_{A} u_{B}\right)^{2}=\delta_{A}^{2} u_{B}^{2}=\delta_{A}^{2} u_{B}$ which is either $\delta_{B}$ or $u_{B}$ so $B$ is full.

"If": Let $A$ and $B$ satisfy (i) and (ii). Let $L^{0}=\left[\delta_{A}, 0_{A}\right), R^{0}=\left(0_{A}, u_{A}\right]$; and $S=L^{0} \cup B \cup R^{0}$. (If $A$ is standard $L^{0}$ is null and references to it below may be ignored.) Order $S$ as in Fig. 2. Define a multiplication "." on $S$ as follows:

(1) For $x, y \in B$ define $x \cdot y=x y$ (the product in $B$ );

(2) for $x \in B, y \in R^{0}$ define $x \cdot y=y \cdot x=x$;

(3) for $x \in B, y \in L^{0}$ define $x \cdot y=x \delta_{B}$ and $y \cdot x=\delta_{B} x$;

(4) for $x, y \in R^{0}$ define $x \cdot y=y \cdot x=x y$ (the product in $A$ with the understanding that if $x y=0_{A}$ in $\left.A, x \cdot y=u_{B}\right)$;

(5) for $x \in R^{0}, y \in L^{0}$ define $x \cdot y=x y$ [and $y \cdot x=y x$ ] (with the understanding that if either product is $0_{A}$ in $A$, we make it $\delta_{B}$ in $S$ );

(6) for $x, y \in L^{0}$ define $x \cdot y=y \cdot x=x y$ (with the understanding that if $x y=0_{A}$ in $A, x \cdot y=\delta_{B}^{2}$.

To verify that "." is associative is mainly routine and utilizes the associativity in $A$ and $B$. Note, however, if we examine $l_{1} \cdot l_{2} \cdot b$ (with $l_{i} \in L^{0}$ and $b \in B$ ), we get $\left(l_{1} \cdot l_{2}\right) \cdot b=\left(l_{1} l_{2}\right) \cdot b=\delta_{B} b$ (if $\left.l_{1} l_{2} \in L^{0}\right)$. While $l_{1} \cdot\left(l_{2} \cdot b\right)=l_{1} \cdot\left(\delta_{B} b\right)$ $=\delta_{B}^{2} b$. But if $B$ is full $\delta_{B}^{2}=\delta_{B}$ and if $A$ is left trivial $l_{1} \cdot l_{2}=\delta_{B}^{2}$ and associativity is preserved.

${ }^{(3)}$ The authors are indebted to the referee for numerous suggestions concerning the statement and proof of this result. 
Note also that the continuity of "." can be verified by checking the (large) number of special cases, and that, in particular, as one (or both) of the factors approaches $\delta_{B}$, the fact that $\lim l_{1} \cdot l_{2}=\delta_{B}^{2}$ depends on the fullness of $B$ or the left triviality of $A$.

Thus $S$ is a semigroup and it is straightforward to verify the following: $u_{A}$ is a unit making $S$ a clan; $B$ is an ideal and a subclan; and $A=S / B$. It remains only to show that the extension is unique. Suppose then that $S^{\prime}$ with multiplication "O" is an extension of $B$ by $A$. We show that "o" agrees with "." in the six classifications above.

(1) Since $B$ is a subclan, $x \circ y=x y$.

(2) $x \circ y=\left(x \circ u_{B}\right) \circ y=x \circ\left(u_{B} \circ y\right)=x \circ u_{B}=x$ (using the relations developed earlier in the proof.)

(3) Let $y=\delta_{A} \circ z$ for some $z \in R^{0}$; then $y \circ x=\delta_{A} \circ(z \circ x)=\delta_{A} \circ x$ by (2). Letting $y$ approach $\delta_{B}$ we get by continuity $\delta_{B} \circ x=\delta_{A} \circ x$. Therefore $y \circ x$ $=\delta_{B} \circ x=\delta_{B} x$. Similarly $x \circ y=x \delta_{B}$.

(4) Since $A=S / B, x \circ y=x y$ unless $x \circ y \in B$. But in that case since $x, y \geqq u_{B}$, we have $x \circ y \geqq u_{B}^{2}=u_{B}$; so $x \circ y=u_{B}$.

(5) Again $x \circ y=x y$ unless $x \circ y \in B$. In that case we let $y=z \circ \delta_{A}$. Now since $x \circ z \geqq u_{B}$ we have $x \circ y=x \circ z \circ \delta_{A} \geqq u_{B} \circ \delta_{A} \geqq u_{B} \circ \delta_{B}=\delta_{B}$.

(6) Again $x \circ y=x y$ unless $x \circ y \in B$. In that case $x \circ y \leqq \delta_{B}^{2}$; but $x$ and $y \geqq \delta_{B}$ implies $x \circ y \geqq \delta_{B}^{2}$. Hence $x \circ y=\delta_{B}^{2}$ completing the proof.

Now for any standard clan $K$ with $k \in K$ we define a collection of clans we call $\mathrm{s}(K, k)$ as follows: Let $p$ be the largest idempotent in $K \leqq k$. Let $\left[p^{\prime}, 0^{\prime}\right]$ be an inverted copy of $[0, p]$. Identify 0 and $0^{\prime}$ and let $S_{1}$ be the interval $\left[p^{\prime}, p\right]$. Extend the multiplication on $[0, p]$ to $S_{1}$ by defining

$$
\begin{aligned}
x^{\prime} \cdot y^{\prime} & =x y \text { and } \\
x^{\prime} \cdot y & =x \cdot y^{\prime}=(x y)^{\prime} .
\end{aligned}
$$

CASE I. If $k=u$, we let $\delta(K, k)$ consist of the clan $S_{1}$.

CASE II. If $k=p<u$, we may, since $[p, u]$ is a standard clan, form $S_{2}$, the extension of $S_{1}$ by $[p, u]$ (using 6.1). Alternatively if $T$ is any left trivial clan with $R=[p, u]$, there is $S_{3}$, the unique extension of $S_{1}$ by $T$. For Case II we let $S(K, k)=\left\{S_{2}\right\} \cup\left\{\right.$ all $\left.S_{3}\right\}$.

CASE III. If $k>p$, let $q$ be first idempotent $>k$. Now $[p, q]$ is a $C$ or $U$-mob. Let $B$ be any base clan with $R=[p, q]$ and $\delta^{2}=k . B$ and $S_{1}$ are matched and $S_{1}$ is full so we may form $S_{4}$, the extension of $S_{1}$ by $B$. If $q<u$, let $T$ be any left trivial clan with $R=[q, u]$. Let $S_{5}$ be the extension of $S_{4}$ by $T$. Alternatively we have $S_{6}$ the extension of $S_{4}$ by $[q, u]$. In Case III we let

$$
\delta(K, k)=\left[\begin{array}{l}
\left\{\text { all } S_{4}\right\} \text { if } q=u \text { or } \\
\left\{\text { all } S_{5}\right\} \cup\left\{\text { all } S_{6}\right\} \text { if } q<u
\end{array}\right] .
$$

Note that in each case $\delta(K, k)$ is a collection of clans having $R=K$ and $\delta^{2}=k$. 
Theorem 6.2. If $S$ is a clan with $\delta^{2} \in R, S \in \mathcal{S}\left(R, \delta^{2}\right)$.

Proof. CASE I, $\delta^{2}=u$. Consider the function $\delta: R \rightarrow L$ defined by $\delta(r)=\delta r$. It is easily seen to be $1-1$, onto, and bicontinuous, and since $\delta(u)=\delta$, we have $L$ is an inverted copy of $R$. Moreover, $\delta(x) \delta(y)=\delta x \delta y=\delta^{2} x y=x y$, and $[\delta(x)] y=\delta x y=\delta(x y)$ so $S$, in this case, is the " $S_{1}$ " in the definition above.

CASE II, $\delta^{2}$ is an idempotent $\prec u$. Let $p=\delta^{2}$ and $\epsilon=\inf \left\{l \mid l^{2}=p\right\}$. Since $(p \epsilon)^{2}=p^{2} \epsilon^{2}=p^{3}=p, p \epsilon=\epsilon$, and, by Case I, $[\epsilon, p]$ is $S_{1}$. If $\epsilon=\delta, S$ is the extension of $[\delta, p]$ by $[p, u]$ and is $S_{2}$. If $\epsilon<\delta$, then $S /[\epsilon, p]$ is left trivial; so $S$ is an $S_{3}$.

CASE III, $\delta^{2}$ is not idempotent. Let $p$ be the first idempotent $\left\langle\delta^{2}, q\right.$ be the first idempotent $>\delta^{2}$, and $\epsilon=\inf \left\{l \mid l^{2}=\delta^{2}\right\}$. Since $(p \epsilon)^{2}=p^{2} \epsilon^{2}=p^{2} \delta^{2}=p^{2}$ $=p$, the interval $[p \epsilon, p]$ is $S_{1}$. Also since $[p, q]$ is a $C$ - or $U$-mob and $q \epsilon=\epsilon$, $[\epsilon, q] /[p \epsilon, p]$ is a base clan by 5.6. Hence $[\epsilon, q]$ is an $S_{4}$. If $q=u, q \delta=\delta$, and $[\delta, q] / \mid p \epsilon, p]$ is a base clan; hence $\delta=\epsilon$ and $S$ is an $S_{4}$. If $q<u$, and $\epsilon=\delta, S$ is the extension of $[\epsilon, q]$ by $[q, u]$ and is an $S_{6}$. If $\epsilon<\delta, S /[\epsilon, q]$ is left trivial and $S$ is an $S_{5}$. So that in any event $S \in \delta\left(R, \delta^{2}\right)$, which was to be shown.

We now define $\mathfrak{N}(K, k)$ for $k \in K$ a standard clan. This definition is similar to that of $s(K, k)$ with the exception of the construction of $S_{1}$ which goes as follows: Let $p$ be the largest idempotent $\leqq k$. Let $f$ be any continuous monotone function of $[0, p]$ onto an interval $J$ with the property that $f^{-1}(t)$ is either a point or a submob of $[0, p]$. Identify 0 and $f(0)$ and let $S_{1}$ be the interval $[f(p), p]$. It is clear that $f$ now satisfies (i) through (iv) of Lemma 2.6 (with $p=u$ and $f(p)=\delta$ ). Define multiplication in $J$ by $t_{1} \cdot t_{2}=f\left[f^{-1}\left(t_{1}\right) f^{-1}\left(t_{2}\right)\right]$. To show "." is well defined (and establish the similarity of this construction with that of $\$ 3)$ we proceed as in Lemma 3.3. Let $a \in f^{-1}\left(t_{1}\right)$ and $b \in f^{-1}\left(t_{2}\right)$. If a and $b$ are unique, there is nothing to show. If not, say $a \neq m=\inf f^{-1}\left(t_{1}\right)$ $=m^{2}$. Then either $a b=m b$ or $b>m$ and $f(a b) \geqq f(m b)=f(m)=f(a) \geqq f(a b)$ so $f(a b)=f(m b)$ and "." is independent of the choice of $a$. In a similar manner we show "." is also independent of $b$, completing the proof. Note that $f$ is now a homomorphism. Define mixed products $r \cdot t=t \cdot r=f\left(f^{-1}(t) r\right)$. By referring to the proof of Theorem 3.2, it is easy to complete the verification that $S_{1}$ is a clan. Note that $f(p)$ is idempotent so $S_{1}$ is full.

CASE I. If $k=u$, we let $\Re(K, k)=\left\{\right.$ all $\left.S_{1}\right\}$.

CASE II. If $k=p \prec u$, then for each $S_{1}$ there is $S_{2}$, the extension of $S_{1}$ by $[p, u]$. There are also left trivial clans, $T$, with $R=[p, u]$; hence there are clans, $S_{3}$, which are extensions of $S_{1}$ by $T$. We let $\Re(K, k)=\left\{\right.$ all $\left.S_{2}\right\} \cup\left\{\right.$ all $\left.S_{3}\right\}$.

CASE III. If $k>p, k \in[p, q]$ a $C$ - or $U$-mob. Let $B$ be any $N$-base clan with $R=[p, q]$ and $k \delta=\delta^{2}$. Let $S_{4}$ be the extension of $S_{1}$ by $B$. If $q \prec u$, let $S_{5}$ be the extension of $S_{4}$ by $T$ (where $T$ is any left trivial clan with $R=[q, u]$ ) or alternatively let $S_{6}$ be the extension of $S_{4}$ by $[q, u]$. We let

$$
\Re(K, k)=\left[\begin{array}{l}
\left\{\text { all } S_{4}\right\} \text { if } q=u \\
\left\{\text { all } S_{5}\right\} \cup\left\{\text { all } S_{6}\right\} \text { if } q \prec u
\end{array}\right] .
$$


Note that in all cases $\mathscr{N}(K, k)$ is a collection of clans each having $L^{2} C L$, $R=K$, and $k \delta=\delta^{2}$.

THEOREM 6.3. If $S$ is a clan with $\delta^{2} \in L$ and $k=\inf \left\{r \mid r \delta=\delta^{2}\right\}$, then $S \in \Re(R, k)$.

Proof. Case I, $\delta=\delta^{2}$. Since $k^{2} \delta=k(k \delta)=k \delta^{2}=k \delta=\delta^{2}, k$ is idempotent. The function $f:[0, k] \rightarrow L$ given by $f(r)=\delta r$ is easily seen to be a continuous homomorphism onto. Moreover, since $S$ is pointed, we have, by Theorem 4.3, $l \cdot r=r \cdot l=r \cdot \delta f^{-1}(l)=\delta \cdot f^{-1}(l) r=f\left(f^{-1}(l) r\right)$. Hence $[\delta, k]$ is an $S_{1}$. If $k=u$, $S$ is an $S_{1}$; if not, $S$ is the extension of $[\delta, k]$ by $[k, u]$ and is an $S_{2}$. Note, incidentally, that whether $k=u$ or not, that $S$ is an $S_{1} \in \Re(R, u)$ whenever $\delta$ is idempotent. This theorem also appears in Clifford [2].

CASE II, $\delta \neq \delta^{2}$ but $k=k^{2}$. Clearly $k \prec u$, and we may consider $S /\left[\delta^{2}, k\right]$ which is easily seen to be a left trivial clan. Now $\left(\delta^{2}\right)^{2}=(k \delta)^{2}=k^{2} \delta^{2}=k^{2} k \delta$ $=k \delta=\delta^{2}$, so that $\left[\delta^{2}, k\right]$ is an $S_{1}$ by Case I and $S$ is an $S_{3}$.

CASE III, $\delta \neq \delta^{2}$ and $k \neq k^{2}$. We have $k \in[p, q]$ a $C$ - or $U$-mob. Let $\epsilon$ $=\inf \left\{l \mid l^{2}=\delta^{2}\right\}$. Since $(q \epsilon)^{2}=q \epsilon^{2}=q \delta^{2}=q k \delta=k \delta=\delta^{2}, q \epsilon=\epsilon$ and $[\epsilon, q]$ is a

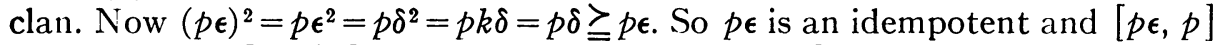
is an $S_{1}$. Now $[\epsilon, q] /[p \epsilon, p]$ is an $N$-base clan, so $[\epsilon, q]$ is an $S_{4}$. If $q=u$, as above, $\epsilon=\delta$ and $S$ is an $S_{4}$. If $q \prec u$ and $\epsilon=\delta, S$ is an extension of $[\epsilon, q]$ by $[q, u]$ and is an $S_{6}$. If $q \prec u$ and $\epsilon \prec \delta$, then $S /[\epsilon, q]$ is left trivial and $S$ is an $S_{5}$. Thus $S \in \Re(R, k)$ completing the proof.

Theorems 6.2 and 6.3 completely characterize clans with a given $R$, but since all standard clans are known, we have characterizations for all clans with zero on an interval.

\section{REFERENCES}

1. A. H. Clifford, Naturally totally ordered commutative semigroups, Amer. J. Math. vol. 76 (1954) pp. 631-646.

2. - Connected ordered topological semigroups with idempotent endpoints. I, Trans. Amer. Math. Soc. vol. 88 (1958) pp. 80-98.

3. W. M. Faucett, Compact semigroups irreducibly connected between two idempotents, Proc. Amer. Math. Soc. vol. 6 (1955) pp. 741-747.

4. - Topological semigroups and continua with cut points, Proc. Amer. Math. Soc. vol. 6 (1955) pp. 748-756.

5. P. S. Mostert and A. L. Shields, On the structure of semigroups on a compact manifold with boundary, Ann. of Math. vol. 65 (1957) pp. 117-143.

6. D. Rees, On semigroups, Proc. Cambridge Philos. Soc., vol. 36 (1940), pp. 387-400.

7. A. D. Wallace, A Note on mobs I, Anais Acad. Brasil. Ci. vol. 24 (1952) pp. 329-334.

8. - The structure of topological semigroups, Bull. Amer. Math. Soc. vol. 61 (1955) pp. 95-112.

Louisiana State University,

Baton Rouge, La. 